\title{
THE STRUCTURE OF $B[c]$ AND EXTENSIONS OF THE CONCEPT OF CONULL MATRIX
}

\author{
H. I. BROWN, ${ }^{1}$ D. R. KERR ${ }^{1}$ AND H. H. STRATTON ${ }^{2}$
}

Introduction. The algebra $\Gamma$ of conservative matrices is partitioned by the ideal $\Psi$ of conull matrices and the set of coregular matrices. This paper is concerned with the problem of extending the concept of conullity from $\Gamma$ to the algebra $B[c]$ of bounded linear operators on the space $c$ of convergent sequences. Since $\Psi$ can be realized as the kernel of a scalar homomorphism $\chi$ on $\Gamma$ (i.e., $\chi$ is a linear functional on $\Gamma$ so that $\chi(A B)=\chi(A) \chi(B)$ for $A, B \in \Gamma)$, one possibility is to consider extensions of $\chi$ in hopes that their kernels will be the natural extensions of $\Psi$. Wilansky [2, p. 250, Question 3] considers two such extensions, $\chi$ and $\rho$, and observes that they both fail to be a scalar homomorphism on $B[c]$. By investigating the algebraic structure of $B[c]$ it is seen that, in a sense, Wilansky's extensions are the only ones possible. For example, in $\S 1$ it is proved that the only subalgebras of $B[c]$ which properly contain $\Psi$ are $\Gamma, \rho_{\perp}$ (the kernel of $\rho$ ), $\Omega$ (the domain of $\rho$ ), and $B[c]$. In $\S 3$ it is proved that $\chi$ is the only nonzero scalar homomorphism on $\Gamma$, that $\rho$ is the only nonzero scalar homomorphism on $\Omega$, and that there are no nonzero scalar homomorphisms on $B[c]$. From these facts we can conclude that $\rho$ is the only extension of $\chi$ to a scalar homomorphism on a subalgebra of $B[c]$. Therefore, if we are to extend the concept of conullity by means of scalar homomorphisms, then $\rho_{\perp}$ becomes the natural definition for conullity in $B[c]$.

Let $M$ designate the multiplicative operators in $B[c]$ (defined below). Unlike the conull-coregular dichotomy in $\Gamma$, the dichotomy of multiplicative and nonmultiplicative matrices cannot be effected by a scalar homomorphism. We show that this behavior is retained in subalgebras of $B[c]$ containing $M \cap \Gamma$. For example, in $\S 2$ it is seen that the only subalgebras of $B[c]$ which properly contain $M \cap \Gamma$ are $\Gamma, M \cap \Omega_{0}$ (defined below), $M \cap \Omega, \Omega, M$, and $B[c]$. Moreover, in $\S 3$ it is proved that $\rho$ and $\chi$ are the only nonzero scalar homomorphisms on any of the above subalgebras of $M$. Hence no one of these algebras can be realized as the kernel of a scalar homomorphism on a larger algebra.

Received by the editors August 19, 1968.

${ }^{1}$ Research partially supported by NSF Grant GP-8199.

2 Research partially supported by NSF Grant GP-8502. 
Definitions, notation, and background. $c$ is the Banach space of convergent sequences $x$ with $\|x\|=\sup \left|x_{i}\right|$. For $x \in c, \lim x$ means $\lim _{i} x_{i}$. Let $e, e^{k}$ for $k \in I^{+}$be those elements of $c$ defined respectively by $e_{i}=1$ for each $i \in I^{+}$and $e_{i}^{k}=\delta_{i k}$ where $\delta_{i k}$ is the Kronecker delta. $B[c]$ is the Banach algebra of bounded linear operators on $c$ with $\|T\|$ $=\sup \{\|T x\|: x \in c$ and $\|x\| \leqq 1\}$. For each $T \in B[c], \chi(T)=\lim T e$ $-\sum_{k} \lim T e^{k}$ and $\chi_{i}(T)=(T e)_{i}-\sum_{k}\left(T e^{k}\right)_{i}$ for each $i \in I^{+},[2, \mathrm{p}$. 241]. Let

$$
\begin{aligned}
\Omega & =\left\{T \in B[c]: \lim _{i} x_{i}(T) \text { exists, }\right\} \\
\Omega_{0} & =\left\{T \in B[c]: \lim _{i} x_{i}(T)=0\right\}, \\
\Gamma & =\{T \in B[c]: T \text { is a matrix }\}, \\
\Psi & =\{T \in \Gamma: \chi(T)=0\}, \\
M & =\{T \in B[c]: \text { For some scalar } \alpha, \lim T x=\alpha \lim x \text { for each } x \in c\} .
\end{aligned}
$$

$\Gamma$ has the equivalent definition $[2$, p. 241]

$$
\left\{T \in B[c]: \chi_{i}(T)=0 \text { for each } i \in I^{+}\right\} \text {. }
$$

We can write each $T \in B[c]$ as follows:

$$
T x=(\lim x) v+B x \quad \text { for } x \in c,
$$

where $v=\left\{\chi_{i}(T)\right\}$, and $B$ is the matrix obtained by restricting $T$ to those elements of $c$ which converge to 0 . This relationship between $T$, $v$, and $B$ is denoted by $T \sim(v, B)$. Further discussion of this representation is to be found in [1].

For $T \in \Omega$ define $\rho(T)=\chi(B)$, where $T \sim(v, B)$. Note that since $T \in \Omega, B \in \Gamma$, and so the definition makes sense.

For $T \in B[c],[T]$ is the matrix representation of the second adjoint of $T$. The entries of [T] will be designated by the upper case letters $T_{i j}$ and are defined as follows: $T_{11}=\chi(T), T_{i 1}=\chi_{i-1}(T)$ for $i>1, T_{1 j}$ $=\lim T e^{j-1}$ for $j>1$, and $T_{i j}=b_{i-1, j-1}$ for $i>1$ and $j>1$, where $T \sim(v, B) .[T]$ is related to $T$ by the equation $T x=J^{-1}[T] J x$ for each $x \in c$, where $J x=\left\{\lim x, x_{1}, x_{2}, \cdots\right\}$. The representation $[T]$ has the advantage that it displays many salient properties of $T$, and will be used extensively in what follows.

For $T \in \Omega$ define $V(T) \in \Omega$ by $V(T)_{11}=\lim \chi_{i}(T), V(T)_{i 1}=\chi_{i-1}(T)$ for $i>1, V(T)_{i j}=0$ otherwise (where $V(T)_{i j}$ are the entries of $[V(T)])$, and define $B(T) \in \Gamma$ by $B(T)_{11}=\rho(T), B(T)_{i 1}=0$ for $i>1$, $B(T)_{i j}=T_{i j}$ otherwise. Note that $T=V(T)+B(T)$. 
The following conventions are made. $E$ always represents the operator $(e, 0) . \Lambda$ always designates a subalgebra of $B[c]$, and every algebra mentioned is assumed to be a subalgebra of $B[c]$. All undesignated entries in a matrix are assumed to be zero. "Scalar homomorphism" means "nonzero scalar homomorphism."

Further properties of $\chi, \rho, \chi_{\perp}, \Omega,[T],(\nu, B)$, etc. are developed in $[1]$.

1. Algebras containing $\Psi$. Results of this section establish that the only subalgebras of $B[c]$ which contain $\Psi$ are $\Psi, \Gamma, \rho_{\perp}, \Omega$, and $B[c]$. We begin with a technical lemma. Let $m$ denote the Banach space of bounded sequences.

1.1. Leмma. If $z \in m$ and $x \in m-c$, then there exists $R \in M \cap \Psi$ so that $z=R x$.

Proof. Let $\alpha$ and $\beta$ be distinct cluster points of $x$ with $\beta \neq 0$. Let $\{n(j)\}$ and $\{k(j)\}$ be disjoint strictly increasing subsequences of $I^{+}$ so that for $j \in I^{+}, x_{n(j)} \neq 0$ and $x_{k(j)} \neq \beta$, and so that $\lim _{j} x_{k(j)}=\alpha$ and $\lim _{j} x_{n(j)}=\beta$. Define the subsequence $\{m(j)\}$ of $I^{+}$by $m(2 j-1)=n(j)$ and $m(2 j)=k(j)$.

We now define five matrices whose product is the desired matrix $R$.

$$
\begin{aligned}
& V: v_{j, m(j)}=1, \\
& U: u_{2 j-1,2 j-1}=1 /(V x)_{2 j-1}, \quad u_{2 j, 2 j}=1 / \beta, \\
& W: w_{j j}=1, \quad w_{2 j, 2 j+1}=w_{2 j-1,2 j+1}=-1, \\
& P: \quad p_{2 j, 2 j}=1 /\left(\left(x_{k(j)} / \beta\right)-1\right), \quad p_{2 j-1,2 j-1}=1 /((\alpha / \beta)-1), \\
& Q: q_{j j}=-q_{j, j+1}=(-1)^{j} z_{j} .
\end{aligned}
$$

Obviously $V, U, W, P$, and $Q$ are all in $M$. Moreover, $Q$ is in $\Psi$ and $\Psi$ is an ideal in $\Gamma$. Therefore, $R=Q P W U V \in M \cap \Psi$. That $z=R x$ is a straightforward computation.

\subsection{Leмma. No proper algebra contains $\chi_{\perp}$.}

Proof. Since $\chi$ is linear, $\chi \perp$ is a maximal subspace of $B[c]$. The result follows since $\chi \perp$ is not an algebra [2, Lemma 3].

1.3. Theorem. $B[c]$ is the only algebra containing $\Psi$ and some element not in $\Omega$.

Proof. Let $\Lambda \supset \Psi$ and let $S \in \Lambda-\Omega$. By Lemma 1.2 it suffices to show that $\Lambda \supset \chi_{\perp}$. By Lemma 1.1 , given $T \in \chi_{\perp}$ we may choose $R \in M \cap \Psi$ so that $\left\{\chi_{i}(T)\right\}=R\left\{\chi_{i}(S)\right\}$. Then $T-R S \in \Psi$ since the 
first column of $[T-R S]$ is zero. Hence $T=T-R S+R S \in \Lambda$, and so $\Lambda$ must be $B[c]$.

Let $B_{c}$ be the set of $T \in B[c]$ such that all columns of [T] are zero except the first which is in $c$, and let $B_{0}$ be the set of those $T$ in $B_{c}$ whose first column converges to zero.

1.4. Lemma. Any algebra containing $\Psi$ and a nonmatrix contains $B_{c}$.

Proof. Let $\Lambda \supset \Psi$ and let $S \in \Lambda-\Gamma$. If $T \in B_{c}$, then

$$
T-\left(\lim _{i} T_{i 1}\right) E \in B_{0} \text {. }
$$

Hence to show that $\Lambda \supset B_{c}$ it suffices to show that $E \in \Lambda$ and that $\Lambda \supset B_{0}$.

Since $S \notin \Gamma, \chi_{k}(S) \neq 0$ for some $k \in I^{+}$. Define $V \in \Psi$ by $v_{k k}=1 / \chi_{k}(S)$. Clearly $V S \in \Lambda$. Define $P \in \Psi$ by $p_{k j}=\left(S e^{j}\right)_{k} / \chi_{k}(S)$ for each $j \in I^{+}$. Then letting $F=V S-P$ we see that $F_{k+1,1}=1$. Thus, by defining $G \in \Psi$ by $G_{i, k+1}=1$ for $i \in I^{+}$, we establish that $E=G F \in \Lambda$.

Finally, if $T \in B_{0}$ define $R \in \Psi$ by $r_{i i}=T_{i 1}$ for $i \in I^{+}$. Then $T$ $=R E \in \Lambda$.

1.5. THEOREM. The only algebras in $\Omega$ which contain $\Psi$ and a nonmatrix are $\Omega$ and $\rho_{\perp}$.

Proof. That $\Omega$ is an algebra can be verified by computing $[S][T]$ for $S, T \in \Omega$. To show that $\rho_{\perp}$ is an algebra we need only show that $S T \in \rho_{\perp}$ whenever $S, T \in \rho_{\perp}$. If $T \in \Omega$, then $[T] \in \Gamma$. Since $\chi$ is multiplicative on $\Gamma$ and since $[S T]=[S][T]$, it remains to show that $\rho(T)$ $=\chi([T])$. But if $T \sim(v, B)$, then

$$
\begin{aligned}
\rho(T)= & \chi(B)=\lim _{n} \sum_{j} b_{n j}-\sum_{j} \lim _{n} b_{n j}=\lim _{n}\left(\chi_{n}(T)+\sum_{j} b_{n j}\right) \\
& -\left(\lim _{n} \chi_{n}(T)+\sum_{j} \lim _{n} b_{n j}\right)=\chi([T]) .
\end{aligned}
$$

Thus, $\rho_{\perp}$ is an algebra.

Now suppose that $\Omega \supset \Lambda \supset \Psi$ and $\Lambda-\Gamma \neq \varnothing$. Then by Lemma 1.4, $\Lambda \supset B_{c}$. Either $\Lambda-\rho_{\perp} \neq \varnothing$ or $\Lambda \subset \rho_{\perp}$.

In the first case, there exists $T \in \Lambda-\rho_{\perp}$. Since $T \in \Omega, T$ $=V(T)+B(T)$ with $B(T) \in \Lambda$. (Indeed, $T \in \Lambda$ and $V(T) \in B_{c} \subset \Lambda$.) Thus if $S \in \Omega$, then $S=V(S)+B(S)$ with $V(S) \in \Lambda$ and $B(S) \in \Lambda$ because $B(S)-(\rho(S) / \rho(T)) B(T) \in \Psi \subset \Lambda$. Hence $S \in \Lambda$, and $\Lambda=\Omega$.

In the second case, if $\Lambda \subset \rho_{\perp}$, then for $S \in \rho_{\perp}, B(S) \in \Psi \subset \Lambda$, and so $S \in \Lambda$. It follows that $\Lambda=\rho_{\perp}$. This completes the proof. 
Noticing that there are no algebras between $\Gamma$ and $\Psi$ we now see that the only subalgebras which contain $\Psi$ are $\Psi, \Gamma, \rho_{\perp}, \Omega$, and $B[c]$.

2. Algebras containing $M \cap \Gamma$. It is not difficult to observe that $T \in M$ if and only if $\lim T e^{i}=0$ for each $i \in I^{+}$, and hence that $M$ is an algebra. Using arguments similar to those in $\$ 1$ we establish that the only algebras containing $M \cap \Gamma$ are $M \cap \Gamma, \Gamma, M \cap \Omega_{0}, M \cap \Omega, \Omega, M$, and $B[c]$. It will be seen from the next section that no one of these algebras can be realized as the kernel of a scalar homomorphism on a larger algebra.

2.1. LEMmA. If an algebra contains $M \cap \Psi$ and a nonmultiplicative operator, then it contains $\Psi$.

Proof. Let $\Lambda$ be such an algebra and let $S \in \Lambda-M$. Then $\lim S e^{k}$ $=b \neq 0$ for some $k \in I^{+}$. Let $T \in \Psi$ and $a_{i}=\lim T e^{i}$ for each $i \in I^{+}$. Define $V \in M \cap \Psi$ by $v_{k j}=a_{j} / b$ for each $j$. The first row of $[S V]$ is the same as the first row of $[T]$. Therefore, $T-S V \in M \cap \Psi$ so that $T=T-S V+S V \in \Lambda$.

2.2. ThEOREM. The only algebras containing $M \cap \Gamma$ and some element not in $\Omega$ are $M$ and $B[c]$.

Proof. Let $\Lambda$ be such an algebra. If $\Lambda-M \neq \varnothing$, then, by Lemma $2.1, \Lambda \supset \Psi$. Hence by Theorem $1.3, \Lambda$ must be $B[c]$. On the other hand, suppose $\Lambda \subset M$. Let $T \in M$ and $S \in \Lambda-\Omega$. Using Lemma 1.1 choose $R \in M \cap \Psi \subset \Lambda$ so that $\left\{\chi_{i}(T)\right\}=R\left\{\chi_{i}(S)\right\}$. Then $R S \in \Lambda$ and $T-R S$ $\in M \cap \Gamma$. Therefore, $T=T-R S+R S \in \Lambda$.

2.3. Lemma. If an algebra contains $M \cap \Gamma$ and a nonmultiplicative operator, then it contains the elements of $\Gamma$ whose columns are constant sequences.

Proof. Let $\Lambda \supset M \cap \Gamma$ and let $S^{\prime} \in \Lambda-M$. Then for some $k \in I^{+}$, $\lim S^{\prime} e^{k} \neq 0$, and so for at most finitely many $i$, say $i(1), \cdots, i(n)$, $\left(S^{\prime} e^{k}\right)_{i(l)}=0$. Define $U \in M \cap \Gamma$ by $\mu_{i(l), k}=1$ for $1 \leqq l \leqq n$. Then $S=U+S^{\prime} \in \Lambda$ and $\left(S e^{k}\right)_{i} \neq 0$ for each $i \in I^{+}$. Define $P \in M \cap \Gamma$ by $p_{i i}=1 /\left(S e^{k}\right)_{i}$ for each $i \in I^{+}$. Then $P S \in \Lambda$. Finally, define $T \in M \cap \Gamma$ by $t_{k j}=\alpha_{j}$, where $\sum\left|\alpha_{j}\right|<\infty$. Then TPS is the matrix whose $j$ th

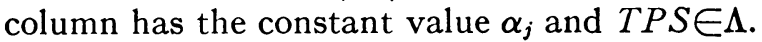

\subsection{Theorem. There are no algebras between $\Gamma$ and $M \cap \Gamma$.}

Proof. Let $\Gamma \supset \Lambda \supset M \cap \Gamma$. If $\Lambda \neq M \cap \Gamma$, then, by Lemma 2.1, $\Lambda \supset \Psi$ and $\Lambda \neq \Psi$. But $\Psi$ is a maximal subalgebra of $\Gamma$, and so we must have $\Lambda=\Gamma$. 
2.5. ThEOREM. Any algebra containing $M \cap \Gamma$ and a nonmatrix contains $M \cap \Omega_{0}$.

Proof. Let $\Lambda$ be such an algebra. As in the proof of Lemma 1.4, there exists $k \in I^{+}$so that $F \in \Lambda$ where $F$ is defined by $F_{k+1,1}=1$. Now let $S \in B_{0}$. Define $S^{\prime} \in M \cap \Gamma$ by $S^{\prime}{ }_{i, k+1}=S_{i 1}$. Then $S=S^{\prime} F \in \Lambda$ and so $\Lambda \supset B_{0}$.

Now let $T \in M \cap \Omega_{0}$. As in Theorem 1.5, $T=V(T)+B(T)$ where $V(T) \in B_{0}$ and $B(T) \in M \cap \Gamma$. Hence $T \in \Lambda$.

2.6. Theorem. Any algebra containing $M \cap \Gamma$ and some nonmatrix not in $M \cap \Omega_{0}$ contains $M \cap \Omega$.

Proof. If $\Lambda$ is such an algebra, then Theorem 2.5 implies $\Lambda \supset B_{0}$. Thus if $T \in M \cap \Omega$, then $V(T) \in B_{c}$ and $B(T) \in M \cap \Gamma$. Since $B_{0}$ is a maximal subspace of $B_{c}$, it suffices to establish the existence of some $S \in \Lambda \cap\left(B_{\mathrm{c}}-B_{0}\right)$.

If $\Lambda-\Omega \neq \varnothing$, the theorem follows from Theorem 2.2 , and if $\Lambda-M$ $\neq \varnothing$ then, by Lemmas 2.1 and $1.4, \Lambda \supset B_{c}$. Therefore, it suffices to consider the case $M \cap \Omega \supset \Lambda$.

Let $S^{\prime} \in \Lambda-M \cap \Omega_{0}$. Then $B\left(S^{\prime}\right) \in M \cap \Gamma$ and so $V\left(S^{\prime}\right) \in \Lambda$. Since $S^{\prime} \notin \Omega_{0}, V\left(S^{\prime}\right) \notin B_{0}$ and so $V\left(S^{\prime}\right)$ is the desired $S$.

2.7. Theorem. Any algebra containing $M \cap \Gamma$ and a nonmultiplicative operator is $\Gamma$ or contains $\Omega$.

Proof. By Lemma 2.3, if $\Lambda$ is such an algebra, then $\Lambda$ contains those elements of $\Gamma$ whose columns are constant sequences. If $\Lambda \subset \Gamma$ then, by Theorem 2.4, $\Lambda=\Gamma$. If $\Lambda \nsubseteq \Gamma$ then, by Lemmas 2.1 and 1.4, $\Lambda \supset B_{c}$ and so, by Theorem 2.6, $\Lambda \supset M \cap \Omega$.

Now let $T \in \Omega$. Define $A \in \Lambda$ by $A_{i, j+1}=\lim T e^{j}$ for $j \in I^{+}$and $i>1$. Then $T-A \in M \cap \Omega \subset \Lambda$ and so $T \in \Lambda$.

3. Scalar homomorphisms on subalgebras of $B[c]$. In this section we will establish that if $\Lambda$ is any one of the nine algebras discussed in $\$ \S 1$ and 2 , and if there exists a scalar homomorphism on $\Lambda$, then it is either $\chi$ or $\rho$.

Let $\theta$ denote the compact operators in $B[c]$. It is known that $A \in \theta \cap \Gamma$ if and only if $\sum_{k}\left|a_{n k}\right|$ converges uniformly with respect to $n$, and that $\Psi \supset \theta \cap \Gamma$ (see [2]).

\subsection{Lemma. There is no scalar homomorphism on $\theta \cap \Gamma$.}

Proof. Let $A \in \theta \cap \Gamma$ and for $j \in I^{+}$let $C^{j} \in \theta \cap \Gamma$ be defined by $C_{i j}^{j}=a_{i j}$ for $i \in I^{+}$. Since $A \in \theta \cap \Gamma, A=\sum_{j=1}^{\infty} C^{j}$ where the convergence of the sum is in the usual norm topology of $\Gamma$. Since $F$ is continuous, 
$F(A)=\sum_{j=1}^{\infty} F\left(C^{j}\right)$. Let $j \in I^{+}$. Define $R, L \in \Gamma$ by $r_{j, j+1}=1$ and $l_{j+1, j}=1$. Then $R^{2}=L^{2}=0$ and $C^{j} R L=C^{j}$. Therefore, $F\left(C^{j}\right)=F\left(C^{j} R L\right)$ $=0$.

3.2. TheOREM. There is no scalar homomorphism on $\Psi$ or on $M \cap \Psi$.

Proof. Let $B \in \Psi$. Define $B^{\prime} \in \theta \cap \Gamma$ by $b_{i j}^{\prime}=\lim _{k} b_{k j}$ for $i, j \in I^{+}$. Then letting $A=B-B^{\prime}$ we get $A \in M \cap \Psi$ (because $\theta \cap \Gamma \subset \Psi$ ), and by Lemma 3.1 $F(A)=F(B)$. It, therefore, suffices to show that there is no scalar homomorphism on $M \cap \Psi$.

Let $A \in M \cap \Psi$ and assume $F(A) \neq 0$ for some scalar homomorphism. Write $A=A_{1}+A_{2}=A_{3}+A_{4}$, where $A_{1}$ (respectively $A_{2}$ ) is the result of replacing the even (respectively odd) rows of $A$ by zeros, and where $A_{3}$ (respectively $A_{4}$ ) is the result of replacing the $4 n-1$ and $4 n$ (respectively $4 n-3$ and $4 n-2$ ) rows of $A$ by zeros. Let $P, Q$, $R, S$ be the elements of $M \cap \Psi$ defined as follows:

$$
\begin{aligned}
1 & =p_{2 n, 2 n-1}=-p_{2 n, 2 n}=q_{2 n-1,2 n}=-q_{2 n-1,2 n+1} \\
& =r_{4 n-1,4 n-3}=r_{4 n, 4 n-2}=-r_{4 n-1,4 n-1}=-r_{4 n, 4 n} \\
& =s_{4 n-3,4 n-1}=s_{4 n-2,4 n}=-s_{4 n-3,4 n+1}=-s_{4 n-2,4 n+2} .
\end{aligned}
$$

Then $Q P A_{1}=A_{1}, P Q A_{2}=A_{2}, S R A_{3}=A_{3}$, and $R S A_{4}=A_{4}$. Since $F(A) \neq 0$, either $F\left(A_{1}\right) \neq 0$ or $F\left(A_{2}\right) \neq 0$. In either case $F(P) \neq 0$ and $F(Q) \neq 0$. Similarly, $F(R) \neq 0$ and $F(S) \neq 0$. Now represent $S$ by $S=S_{1}+S_{2}$, where $S_{1}$ (respectively $S_{2}$ ) is the result of replacing the $4 n-2$ (respectively $4 n-3$ ) rows of $S$ by zeros. Since $F(S) \neq 0$, either $F\left(S_{1}\right) \neq 0$ or $F\left(S_{2}\right) \neq 0$. Either case yields a contradiction since $S_{1} P$ $=0=S_{2} Q$. Hence, $F(A) \neq 0$ is impossible.

3.3. CoROllary. $\chi$ is the only scalar homomorphism on $\Gamma$ and on $M \cap \Gamma$.

\subsection{THEOREM. $\rho$ is a scalar homomorphism on $\Omega$.}

Proof. This result follows from the fact that $\rho_{\perp}$ is an algebra (Theorem 1.5) and from [3, Lemma 3, p. 254].

3.5. TheOREM. $\chi$ and $\rho$ are the only possible scalar homomorphisms on any algebra in $\Omega$ which contains either $\Psi$ or $M \cap \Gamma$.

Proof. Let $\Lambda$ be such an algebra. As shown in $\$ \$ 1$ and $2, \Lambda$ must be one of seven possible algebras. It follows that for any $T \in \Lambda, V(T) \in \Lambda$ and $B(T) \in \Lambda$. Therefore, for any scalar homomorphism $F$ on $\Lambda, F(T)$ $=F(V(T))+F(B(T))$. Since $\Lambda \subset \Omega,\{B(T): T \in \Lambda\}$ is a subalgebra of $\Gamma$ containing either $\Psi$ or $M \cap \Gamma$. Therefore, by Theorem 2.4, Corol- 
lary 3.3, and the fact that there are no subalgebras between $\Gamma$ and $\Psi, \quad F(B(T))=\chi(B(T))=\rho(T)$. Moreover, since $(V(T))^{2}$ $=\left(\lim _{i} \chi_{i}(T)\right) V(T)$, either $F(V(T))=0$ or $F(V(T))=\lim _{i} \chi_{i}(T)$. Therefore, either $F(T)=\rho(T)$ or $F(T)=\lim _{i} \chi_{i}(T)+\rho(T)=\chi(T)$.

3.6. COROLlARY. $\chi$ and $\rho$ are the only scalar homomorphisms on $M \cap \Omega$ and on $M \cap \Omega_{0}$, and, in fact, $\chi=\rho$ on $M \cap \Omega_{0}$.

3.7. LEMмA. If $E \in \Lambda$ and if $F(E) \neq 0$ for some scalar homomorphisms $F$ on $\Lambda$, then $F(T)=\lim T$ e for every $T \in \Lambda$.

Proof. Since $E^{2}=E, F(E)=1$. If $T \in \Lambda$, then $V(E T)=\chi(T) E$ and $B(E T)=B \in \Lambda$ where $b_{i j}=\lim T e^{i}$ for $i, j \in I^{+}$. Therefore, $F(T)$ $=\chi(T)+F(B)$. But $F(B)=F(B E)=\sum_{i=1}^{\infty} \lim T e^{i}$, and so (by the definition of $\chi) F(T)=\lim T e$.

3.8. THEOREM. $\rho$ is the only scalar homomorphism on $\Omega$ and there is no scalar homomorphism on $\rho_{\perp}$.

Proof. This is an immediate consequence of Theorem 3.5 and Lemma 3.7.

3.9. THEOREM. $\chi$ is the only scalar homomorphism on $M$ and there is no scalar homomorphism on $B[c]$.

Proof. It is clear that $\chi$ is a scalar homomorphism on $M$ but not on $B[c]$. By noting that $E \in M$ and that $\chi(T)=\lim T e$ for $T \in M$ but not for all $T \in B[c]$, it suffices, by Lemma 3.7 , to show that $F(E) \neq 0$ for any scalar homomorphism $F$ on $M$ or on $B[c]$.

Let $I$ denote the identity operator. Then $F(I)=1$. Consider the following operators $P, Q, R, S$ in $M$.

$$
\begin{aligned}
P_{2 n, 1} & =Q_{2 n+3,1}=R_{2 n, 1}=S_{2 n+3,1}=-1 ; \\
P_{2 n, 2 n} & =Q_{2 n+3,2 n+3}=R_{2 n, 2 n+1}=S_{2 n+3,2 n+2}=1 .
\end{aligned}
$$

Then $P+Q=I-E$ and so if $F(E)=0$, either $F(P) \neq 0$ or $F(Q) \neq 0$. However, $R S P=P, R P=0, S R Q=Q$, and $S Q=0$, so that $F(P)=0$ and $F(Q)=0$. This contradiction establishes the theorem.

\section{REFERENCES}

1. J. P. Crawford, Transformations in Banach spaces with applications to summability theory, Ph.D. dissertation, Lehigh University, Bethlehem, Pa., 1966.

2. A. Wilansky, Topological divisors of zero and Tauberian theorems, Trans. Amer. Math. Soc. 113 (1964), 240-251.

3. —, Functional analysis, Blaisdell, New York, 1964.

State University of New York at Albany 